TP Periodica Polytechnica

Mechanical Engineering

60(4), pp. 209-219, 2016

DOI: 10.3311/PPme.8707

Creative Commons Attribution (i)

RESEARCH ARTICLE

\section{Free Vibration Analysis of Functionally Graded Nanobeams Based on Different Order Beam Theories Using Ritz Method}

\author{
Abbes Elmeiche ${ }^{1}$, Abdelkader Megueni ${ }^{*}$, Abdelkader Lousdad ${ }^{1}$
}

Received 22 September 2015; accepted after revision 02 May 2016

\begin{abstract}
This paper presents the fundamental frequency analysis of functionally graded ( $F G$ ) nanobeams using Ritz method subjected to different sets of boundary conditions. The vibration analysis is based on the classical, the first-order and different higher-order shear deformation beam theories while including rotary inertia. The material properties of FG nanobeams are assumed to vary through the thickness according to the power-law exponent form. Based on the nonlocal constitutive relations of Eringen, the frequencies equations are obtained by the weak forms of the governing differential equations. In this study, the effects of material distribution, nonlocal parameter, beam theories, slenderness ratios and boundary conditions on the fundamental frequency are discussed. The analysis is validated by comparing the obtained results with the available results from the existing literature.
\end{abstract}

\section{Keywords}

vibration analysis, functionally graded nanobeams, Ritz method, shear deformation, nonlocal parameter

\footnotetext{
${ }^{1}$ Laboratory of Solids and Structures Mechanics, Faculty of Technology, University of Sidi-Bel Abbes, 22000, Algeria

*Corresponding author, e-mail: a_megueni@yahoo.fr
}

\section{Introduction}

Metal and natural materials were commonly used by human since epochs. by the time, the need to produce special products for special condition led to the need to develop special materials, where rose what is called today composite materials, these materials have significant advantages over ordinary materials, they considerably increased the performance of the structure (beam, plate, tube, etc...), these multi layered products are a combination of two or more materials with different properties to withstand specific conditions but unfortunately the effect of some severe conditions such as heat and pressure onto materials with different physical properties induce to a high level of stress concentrations at the interfaces between the altered layers. Research for developing new materials with more specific properties has not stopped, in the late of the $80 \mathrm{~s}$ a group of scientists succeeded to discover a way to gather particles of a structure according to a special method leading to a very specific product with a very specific properties that vary continuously as a known function of the spatial position, they called them Functionally Graded Materials FGM, as their name describe, they are materials usually associated with particulate composite where the volume fraction of particles varies in one or several directions.

The initial development of FGMs is designed to serve as a thermal barrier [1]. Typically, these materials are made from a mixture of metal and ceramic, or a combination of materials. Where the ceramic component provides high temperature resistance due to its low thermal conductivity.

Recently, researches on understanding the dynamic behavior of structural elements with FGMs is increasing and FGMs have known a large expansion in almost all domains of the industry and are used in very different applications of engineering such as in automotive, aerospace, defense industries, and more recently in, electronics, nuclear reactors and biomedical.

In order to properly understand and control the material properties it is very important to analyze and study the effect of free vibrations on mechanical systems, the knowledge of fundamental frequencies allows us to avoid resonances by controlling the frequencies of the affecting forces [2]. 
Many theory are applied to study and describe beams behavior, Euler-Bernoulli beam or classical Beam theory (CBT) is one of the first well-known theory, in this theory the transverse shear deformation is neglected because it assume that the median planes are perpendicular and straight to the section of the beam after bending. But this theory is applied only for thin beams and does not provide specific solutions for thick beams. One other famous beam theory is that Timoshenko beams or first-order shear deformation theory (FSDBT), in which straight lines perpendicular to the median plane before bending does not remain perpendicular to the median plane after bending, the stress distribution of transverse shear relative to the coordinates of thickness is assumed to be constant. Thus, a shear correction factor is required to compensate this assumption. However, this theory provides satisfactory results and is very effective to study the behavior of beams. Several higher order shear deformation theories have been developed in the last years considering warping sections and satisfying the zero transverse shear stress state of the upper and lower fibers of the cross section without a shear correction factor. The well-known higher-order beam theories are Parabolic Shear Deformation Beam Theory (PSDBT) [3], Trigonometric Shear Deformation Beam Theory (TSDBT) [4], Hyperbolic Shear Deformation Beam Theory (HSDBT) [5], Exponential Shear Deformation Beam Theory (ESDBT) [6], and A New Shear Deformation Beam Theory (ASDBT) [7].

Many researchers are interested on the basis of molecular dynamics and continuum mechanics. The nonlocal theory of Eringen [8-11], which is one of continuum mechanics load size models, is widely used. Wang et al. [12] concerned with the use of the Timoshenko beam model for free vibration analysis of multi-walled carbon nanotubes by using differential quadrature method. Reddy [13] applies the Nonlocal theories for bending, buckling and vibration of Euler-Bernoulli, Timoshenko, Reddy and Levinson beams theories. Lu et al. [14] studied nonlocal beam models of wave properties of single and double walled carbon nanotubes. Reddy and Pang [15] reformulated theories of Euler-Bernoulli and Timoshenko for the analysis of carbon nanotubes using differential relationship Eringen nonlocal model. Aydogdu [16] proposed a generalized nonlocal beam theory to study bending, buckling, and free vibration of nanobeams based on Eringen model. Pradhan and Murmu [17] developed a single nonlocal beam model to investigate the bending and vibration characteristics of a nanocantilever beam. Ansari et al. [18] derived the governing partial differential equation for a uniform rotating beam incorporating the nonlocal scale effects. Thai and Vo [19] applied a sinusoidal theory of non-local shear deformation. Eltaher et al. [20] studied the free vibration nanobeams using the finite element method. O. Rahmani et al. [21] examined the size effect on vibration of functionally graded nanobeams based on nonlocal Timoshenko beam theory.
Direct resolution of complex equation system is difficult. A typical approach is to seek a solution by approximating the displacement field from several functions that satisfy the boundary conditions. This is called the Ritz method. Algebraic polynomials functions have been employed on the basis of different beam theories. It is interesting to note that this technique has not been used by researchers to study the vibration problems of FG nanobeams.

The objective of this work is to present an analytical model of the fundamental frequency of functionally graded (FG) nanobeams using Ritz method subjected to different sets of boundary conditions. The vibration analysis is according to all beams theories, CBT, FSDBT, PSDBT, HSDBT, TSDBT, ESDBT and ASDBT, while including rotator inertia. The material properties of FG nanobeams are assumed to vary through the thickness according to the power-law exponent form. Based on the nonlocal constitutive relations of Eringen, the system of equations of motion are derived using virtual work's principle. The frequencies equations are obtained by the weak forms of the governing differential equations where the displacement components of the nanobeam cross-sections are expressed in a series of simple algebraic polynomials. The numerical results, such as fundamental frequencies, are illustrated in graphical and tabular form. The analysis is validated by comparing the obtained results with the available results from the existing literature. In this study, the effects of material distribution, nonlocal parameter, beam theories, slenderness ratios, and boundary conditions on the fundamental frequency are discussed.

\section{Functionally graded materials}

A straight FG nanobeam of length $\mathrm{L}$, width $\mathrm{b}$ and thickness $\mathrm{h}$, with Cartesian coordinate system $(\mathrm{O}, \mathrm{x}, \mathrm{y}, \mathrm{z})$ having the origin at $\mathrm{O}$ is considered, as shown in Fig. 1.

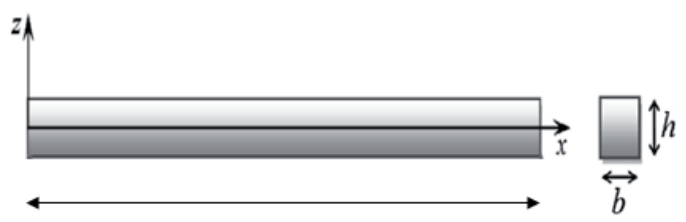

Fig. 1 Schematic of the FG nanobeam

We suppose that the effective material properties FG nanobeam i.e., Young's modulus $(E)$, Poisson's ratio $(v)$ and mass density $(\rho)$, vary along the thickness direction (in the $\mathrm{z}$ direction) according to a function of the volume fractions of the constituents.

Based on to the rule of mixture, the effective material properties $(P)$ can be expressed as:

$$
P=P_{U} V_{U}+P_{L} V_{L}
$$


Where:

$P_{U}, P_{L}, V_{U}$ and $V_{L}$ are the corresponding material properties and the volume fractions of the upper and the lower surfaces of the nanobeam related by:

$$
V_{U}+V_{L}=1
$$

In this study the effective material properties of the FG nanobeam are defined by the power-law form introduced by Wakashima et al. [22]. The volume fraction of the upper constituent is assumed to be given by:

$$
V_{U}=\left(\frac{z}{h}+\frac{1}{2}\right)^{k}
$$

$k$ is the power-law exponent $(0 \leq k \leq \infty)$ which determines the material variation profile through the thickness of the namobeam as shown in the following Fig. 2 .

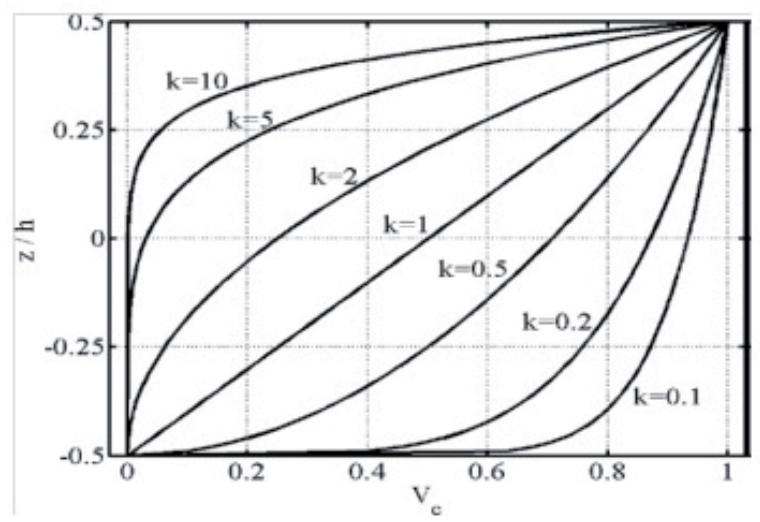

Fig. 2 Power low variation of the volume fraction Vc of the ceramic constituent through the thickness of the FG nanobeam

Using Eqs. (1), (2) and (3), the effective material properties of the FG nanobeam can be given as:

$$
P(z)=\left(P_{U}-P_{L}\right)\left(\frac{z}{h}+\frac{1}{2}\right)^{k}+P_{L}
$$

\section{Nonlocal beam theory}

According to the nonlocal elasticity theory, the stress at a given point depends on the strains of the whole continuum [8]. This assumption may be written as:

$$
\left[1-\mu \nabla^{2}\right] \bar{\sigma}=\bar{C}: \bar{\varepsilon}
$$

Where $\bar{\sigma}$ is the stress tensor, $\bar{C}$ is the Hookean elasticity tensor, and $\bar{\varepsilon}$ is the strain tensor. The symbols $\nabla^{2}$ are the Laplacian operator and double dot tensor product. The nonlocal parameter $\mu=\left(e_{0} a\right)^{2}$ is a scale factor that depends on the material and geometric features. The coefficient $e_{0}$ is estimated such that the non local elasticity matches the atomistic lattice models, and $a$ is the so called internal characteristic lengths [8] and [23]. Thus, the general nonlocal constitutive relation for nanobeams takes the following form:

$$
\left[\begin{array}{l}
\sigma_{x x} \\
\sigma_{x z}
\end{array}\right]-\mu \frac{\partial^{2}}{\partial x^{2}}\left[\begin{array}{l}
\sigma_{x x} \\
\sigma_{x z}
\end{array}\right]=\left[\begin{array}{cc}
Q_{11} & 0 \\
0 & Q_{55}
\end{array}\right]\left\{\begin{array}{l}
\varepsilon_{x x} \\
\gamma_{x z}
\end{array}\right\}
$$

The reduced elastic constants are defined as follows:

$$
Q_{11}=\frac{E(z)}{\left(1-v(z)^{2}\right)} \text { and } Q_{55}=\frac{E(z)}{2(1+v(z))}
$$

where is the elasticity modulus, $v$ is the Poisson's ratio, $\sigma_{x x}$ is the axial normal stress, $\sigma_{x z}$ is the shear stress, $\varepsilon_{x x}$ is the axial strain and $y_{x z}$ is the shear strain. If the nonlocal parameter is zero, we obtain the constitutive relations of the classical beam theories.

\section{Mathematical modeling}

Based on the general shear deformation theory, the axial displacement $u$, and the transverse displacement of any point of the beam $w$ are given as:

$$
\begin{gathered}
u(x, z, t)=u_{0}(x, t)-z \frac{\partial w_{0}(x, t)}{\partial x}+f(z) \varphi_{0}(x, t) \\
v(x, z, t)=0(7) \\
w(x, z, t)=w_{0}(x, t)
\end{gathered}
$$

Where $u_{0}$ and $w_{0}$ represent the axial and the transverse displacement of any point on the neutral axis respectively, while $\varphi_{0}$ is an unknown function that represents the effect of transverse shear strain on the neutral axis. $f(z)$ is the shape function which characterizes the transverse shear and stress distribution along the thickness of the beam. Different beam theories can be obtained by choosing as follows:

$C B T: f(z)=0$

FSDBT $: f(z)=z$

PSDBT $: f(z)=z\left(1-\frac{4 z^{2}}{3 h^{2}}\right)$

TSDBT $: f(z)=\frac{h}{\pi} \sin \left(\frac{\pi z}{h}\right)$

$H S D B T: f(z)=h \sinh \left(\frac{z}{h}\right)-z \cosh \left(\frac{1}{2}\right)$

ESDBT $: f(z)=z e^{-2(z / h)^{2}}$

$A S D B T: f(z)=z \alpha^{-2(z / h)^{2} / \ln \alpha}$ with $\alpha=3$

The strain-displacement relations of the general beam theories are given by:

$$
\begin{gathered}
\varepsilon_{x x}=\frac{\partial u(x, z, t)}{\partial x}=\frac{\partial u_{0}}{\partial x}-z \frac{\partial^{2} w_{0}}{\partial x^{2}}+f(z) \frac{\partial \varphi_{0}}{\partial x} \\
\gamma_{x z}=\frac{\partial u(x, z, t)}{\partial z}+\frac{\partial w(x, z, t)}{\partial x}=\frac{d f(z)}{d z} \varphi_{0}
\end{gathered}
$$


The governing equations will be obtained by applying virtual work principle:

$$
\begin{aligned}
\delta W_{i n t} & =\int_{V} \sigma_{i j} \delta \varepsilon_{i j} d V=\int_{V} \sigma_{x x} \delta \varepsilon_{x x} d V+\int_{V} \sigma_{x z} \delta \gamma_{x z} d V \\
\delta W_{i n t} & =\int_{0}^{L} N^{C} \frac{\partial \delta u_{0}}{\partial x} d x-\int_{0}^{L} M^{C} \frac{\partial^{2} \delta w_{0}}{\partial x^{2}} d x \\
& +\int_{0}^{L} M^{s d} \frac{\partial \delta \varphi_{0}}{\partial x} d x+\int_{0}^{L} Q \delta \varphi_{0} d x
\end{aligned}
$$

Where $N^{C}, M^{C}, M^{\text {sd }}$ and $Q$ are the stress resultants defined as:

$$
\left(N^{c}, M^{c}, M^{s d}\right)=\int_{A} \sigma_{x x}(1, z, f(z)) d A \text { and } Q=\int_{A} \sigma_{x x} \frac{d f(z)}{d z} d A
$$

The resultants denoted with a superscript ' $c$ ' are the conventional ones of the classical beam theories, where as the remaining ones with superscript ' $s d$ ' are additional quantities incorporating the shear deformation effects. By substituting the stress-strain relations into the definitions of the force and the moment resultants of the present theory, the following constitutive equations are obtained:

$$
\left[\begin{array}{c}
N^{c} \\
M^{c} \\
M^{s d}
\end{array}\right]=\left[\begin{array}{lll}
A_{11} & B_{11} & E_{11} \\
B_{11} & D_{11} & F_{11} \\
E_{11} & F_{11} & H_{11}
\end{array}\right]\left[\begin{array}{c}
\frac{\partial u_{0}}{\partial x} \\
-\frac{\partial^{2} w_{0}}{\partial x^{2}} \\
\frac{\partial \varphi_{0}}{\partial x}
\end{array}\right] \text { and } Q=\left[A_{55}\right]\left[\varphi_{0}\right]
$$

The extensional, coupling, bending and transverse shear rigidities are given as follows:

$$
\begin{gathered}
\left(A_{11}, B_{11}, D_{11}\right)=b \int_{-\frac{h}{2}}^{+\frac{h}{2}} Q_{11}\left(1, z, z^{2}\right) d z \\
\left(E_{11}, F_{11}, H_{11}\right)=b \int_{-\frac{h}{2}}^{+\frac{h}{2}} Q_{11} f(z)(1, z, f(z)) d z \\
A_{55}=k_{s} \cdot b \int_{-\frac{h}{2}}^{+\frac{h}{2}} Q_{55}\left[\frac{d f(z)}{d z}\right]^{2} d z \\
\left(k_{s} \text { is the shear correction factor }\right)
\end{gathered}
$$

The work of the external forces when the effect of rotary inertia is taken into consideration is written as follows:

$$
\begin{aligned}
\delta W_{e x t} & =\int_{V} \rho(z) \ddot{u} \delta u d V+\int_{V} \rho(z) \ddot{w} \delta w d V \\
\delta W_{e x t} & =\int_{0}^{L}\left(I_{1} \frac{\partial^{2} u_{0}}{\partial t^{2}}-I_{2} \frac{\partial^{3} w_{0}}{\partial x \partial t^{2}}+I_{3} \frac{\partial^{2} \varphi_{0}}{\partial t^{2}}\right) \delta u_{0} d x \\
& -\int_{0}^{L}\left(I_{1} \frac{\partial^{2} u_{0}}{\partial t^{2}}-I_{4} \frac{\partial^{3} w_{0}}{\partial x \partial t^{2}}+I_{5} \frac{\partial^{2} \varphi_{0}}{\partial t^{2}}\right) \frac{\partial \delta w_{0}}{\partial x} d x \\
& +\int_{0}^{L}\left(I_{3} \frac{\partial^{2} u_{0}}{\partial t^{2}}-I_{5} \frac{\partial^{3} w_{0}}{\partial x \partial t^{2}}+I_{6} \frac{\partial^{2} \varphi_{0}}{\partial t^{2}}\right) \delta \varphi_{0} d x+\int_{0}^{L} I_{1} \frac{\partial^{2} w_{0}}{\partial t^{2}} \delta w_{0} d x
\end{aligned}
$$

Such as:

$$
\left[I_{1}, I_{2}, I_{3}, I_{4}, I_{5}, I_{6}\right]=b \int_{-\frac{h}{2}}^{+\frac{h}{2}} \rho(z)\left[1, z, f(z), z^{2}, z f(z), f(z)^{2}\right] d x
$$

Using principle of virtual work, following governing equations of the beam are obtained as:

$$
\frac{\partial N^{c}}{\partial x}=I_{1} \frac{\partial^{2} u_{0}}{\partial t^{2}}-I_{2} \frac{\partial^{3} w_{0}}{\partial x \partial t^{2}}+I_{3} \frac{\partial^{2} \varphi_{0}}{\partial t^{2}}
$$

$$
\begin{gathered}
\frac{\partial^{2} M^{c}}{\partial x^{2}}-I_{1} \frac{\partial^{2} w_{0}}{\partial t^{2}}=I_{2} \frac{\partial^{3} u_{0}}{\partial x \partial t^{2}}-I_{4} \frac{\partial^{4} w_{0}}{\partial x^{2} \partial t^{2}}+I_{5} \frac{\partial^{3} \varphi_{0}}{\partial x \partial t^{2}}+I_{3} \frac{\partial^{2} \varphi_{0}}{\partial t^{2}} \\
\frac{\partial M^{s d}}{\partial x}-Q=I_{3} \frac{\partial^{2} u_{0}}{\partial t^{2}}-I_{5} \frac{\partial^{3} w_{0}}{\partial x \partial t^{2}}+I_{6} \frac{\partial^{2} \varphi_{0}}{\partial t^{2}}
\end{gathered}
$$

For Ritz method, the amplitudes of vibration are expanded in terms of algebraic polynomial functions by the following series.

$$
\begin{aligned}
& u_{0}(x, t)=\sum_{j=1}^{n}\left[\varphi_{j}(x) u_{j}\right] e^{i \omega t} \\
& w_{0}(x, t)=\sum_{k=1}^{n}\left[\psi_{k}(x) w_{k}\right] e^{i \omega t} \\
& \varphi_{0}(x, t)=\sum_{p=1}^{n}\left[\Phi_{p}(x) \varphi_{p}\right] e^{i \omega t}
\end{aligned}
$$

Where $u_{j}, w_{k}$ and $\varphi_{p}$ are the unknown constant coefficients to be determined. $\omega$ is the natural frequency of the FG nanobeam and $\varphi_{j}, \psi_{k}$ and $\Phi_{p}$ are the admissible functions, which must satisfy the essential boundary conditions and can be represented as:

$$
\begin{aligned}
& \varphi_{j}(x)=(L-x)^{q 0} x^{(j+p 0)-1} \\
& \psi_{k}(x)=(L-x)^{q 0} x^{(k+p 0)-1} \Phi_{p}(x)=(L-x)^{q 0} x^{(p+p 0)-1}
\end{aligned}
$$

Where $n$ is the number of polynomials involved in the admissible functions and $p_{0}, q_{0}$ as per the six boundary conditions as stated in Table 1.

Table 1 Admissible function indices for different boundary conditions

\begin{tabular}{lll}
\hline BCs & $\boldsymbol{p}_{\mathbf{0}}$ & $\boldsymbol{q}_{\mathbf{0}}$ \\
\hline $\mathrm{C}-\mathrm{C}$ & 2 & 2 \\
$\mathrm{C}-\mathrm{S}$ & 2 & 1 \\
$\mathrm{C}-\mathrm{F}$ & 2 & 0 \\
$\mathrm{~S}-\mathrm{S}$ & 1 & 1 \\
$\mathrm{~S}-\mathrm{F}$ & 1 & 0 \\
$\mathrm{~F}-\mathrm{F}$ & 0 & 0 \\
\hline
\end{tabular}

Using principle of virtual work, following governing equations of the nanobeam are obtained as: 


$$
\begin{gathered}
\frac{\partial N^{c}}{\partial x}-\mu\left(I_{1} \frac{\partial^{4} u_{0}}{\partial x^{2} \partial t^{2}}-I_{2} \frac{\partial^{5} w_{0}}{\partial x^{3} \partial t^{2}}+I_{3} \frac{\partial^{4} \varphi_{0}}{\partial x^{2} \partial t^{2}}\right) \\
-\left(A_{11} \frac{\partial^{2} u}{\partial x^{2}}-B_{11} \frac{\partial^{3} w_{0}}{\partial x^{3}}+E_{11} \frac{\partial^{2} \varphi_{0}}{\partial x^{2}}\right)=0 \\
-\frac{\partial^{2} M^{c}}{\partial x^{2}}-\mu\left(I_{2} \frac{\partial^{5} u_{0}}{\partial x^{3} \partial t^{2}}-I_{4} \frac{\partial^{6} w_{0}}{\partial x^{4} \partial t^{2}}+I_{5} \frac{\partial^{5} \varphi_{0}}{\partial x^{3} \partial t^{2}}+I_{1} \frac{\partial^{4} w_{0}}{\partial x^{2} \partial t^{2}}\right) \\
-\left(B_{11} \frac{\partial^{3} u_{0}}{\partial x^{3}}-D_{11} \frac{\partial^{4} w_{0}}{\partial x^{4}}+F_{11} \frac{\partial^{3} \varphi_{0}}{\partial x^{3}}\right)=0 \\
\frac{\partial M^{s d}}{\partial x}-Q-\mu\left(I_{3} \frac{\partial^{4} u_{0}}{\partial x^{2} \partial t^{2}}-I_{5} \frac{\partial^{5} w_{0}}{\partial x^{3} \partial t^{2}}+I_{6} \frac{\partial^{4} \varphi_{0}}{\partial x^{2} \partial t^{2}}\right) \\
-\left(E_{11} \frac{\partial^{2} u}{\partial x^{2}}-F_{11} \frac{\partial^{3} w_{0}}{\partial x^{3}}+H_{11} \frac{\partial^{2} \varphi_{0}}{\partial x^{2}}\right) \\
-\left(A_{11} \frac{\partial^{2} u}{\partial x^{2}}-B_{11} \frac{\partial^{3} w_{0}}{\partial x^{3}}+E_{11} \frac{\partial^{2} \varphi_{0}}{\partial x^{2}}\right)+A_{55} \varphi_{0}=0
\end{gathered}
$$

The weak forms of differential Eqs. (20), (21), and (22) are obtained by integration of these equations with the weighted function respectively $\varphi_{i}(x), \quad \psi_{i}(x)$ and $\Phi_{i}(x)(i=1,2, \ldots)$ which must satisfy the boundary conditions.

These weak forms are a generalized eigenvalue problem and that written as the following form:

$$
\left([K]-\omega^{2}[M]\right)\{q\}=0
$$

Where $[K]$ and $[M]$ are the stiffness and inertia matrices respectively, their order is $[3 n \times 3 n],\{q\}$ is the column vector of unknown coefficients of Eq. (23) of order $\{3 n \times 1\}$. The eigenvalue $\omega$ are solution of the following equation:

$$
\operatorname{det}\left([K]-\omega^{2}[M]\right)=0
$$

\section{Numerical results and discussion}

The fundamental frequencies of FG nanobeams subjected to different sets of boundary conditions , Clamped-Clamped (C-C), Clamped-Simply (C-S), Simply-Simply (S-S), and Clamped-Free $(\mathrm{C}-\mathrm{F})$ are presented with varying nonlocal parameter $(\mu)$, material distribution $(k)$, beam theories, slenderness ratio $(\mathrm{L} / \mathrm{h})$ and boundary conditions.

The shear correction factor is considered as $\mathrm{ks}=5 / 6$ for FSDBT. Fundamental frequencies are non-dimensionalized according to the following relation:

$$
\varpi=\omega L^{2} \sqrt{\frac{\rho_{U} A}{E_{U} I}}
$$

Where:

$I=b h^{3} / 12$ is the moment of inertia of the cross section of the nanobeam.

In this study the FG nanobeams are made of a ceramic and metal mixture whose the properties varies through the thickness according to power-law. The upper side of the nanobeam $(\mathrm{z}=+\mathrm{h} / 2)$ is pure ceramic (Alumina), while the lower side of the nanobeam $(\mathrm{z}=-\mathrm{h} / 2)$ is pure metal (Aluminum). The material properties which used in the present study are given in Table 2 and the thickness (h) of FG nanobeam is $1 \mathrm{~nm}$.

Table 2 Material properties of the FGM constituents

\begin{tabular}{cccc}
\hline Properties & Unit & Aluminium $(\mathrm{Al})$ & Alumina $\left(\mathrm{Al}_{2} \mathrm{O}_{3}\right)$ \\
\hline$E$ & $\mathrm{GPa}$ & 70 & 380 \\
$\rho$ & $\mathrm{kg} / \mathrm{m}^{3}$ & 2700 & 3800 \\
$v$ & - & 0.23 & 0.23 \\
\hline
\end{tabular}

In Table 3 and Fig. 3, the convergence studies for first fundamental frequency of $\mathrm{C}-\mathrm{C}$ FG nanobeam are performed with various number of polynomials (n) using different beam theories with $\mathrm{k}=1$ and $\mu=3$ for $\mathrm{L} / \mathrm{h}=10$. It is seen that in the Ritz method, the increased number (n) in the displacement functions plays a major role in the convergence of the frequencies. The numerical accuracy of fundamental frequency is satisfactory when the number of terms in the displacement functions is set to 16 .

Table 3 Convergence study for C-C FG nanobeam with $\mathrm{k}=1$ and $\mathrm{mu}=3$ for $\mathrm{L} / \mathrm{h}=10$

\begin{tabular}{ccccc}
\hline $\mathrm{n}$ & CBT & FSDBT & PSDBT & ESDBT \\
\hline 2 & 16.3237 & 16.3237 & 16.3237 & 16.3237 \\
4 & 15.1393 & 14.6080 & 14.6087 & 14.6120 \\
6 & 15.0045 & 14.4087 & 14.4096 & 14.1434 \\
8 & 14.9450 & 14.3235 & 14.3246 & 14.3286 \\
10 & 14.9147 & 14.2800 & 14.2814 & 14.2856 \\
12 & 14.8971 & 14.2548 & 14.2564 & 14.2608 \\
14 & 14.8860 & 14.2374 & 14.2410 & 14.2443 \\
16 & 14.8785 & 14.2338 & 14.2360 & 14.2406 \\
18 & 14.8732 & 14.2325 & 14.2315 & 14.2375 \\
\hline
\end{tabular}

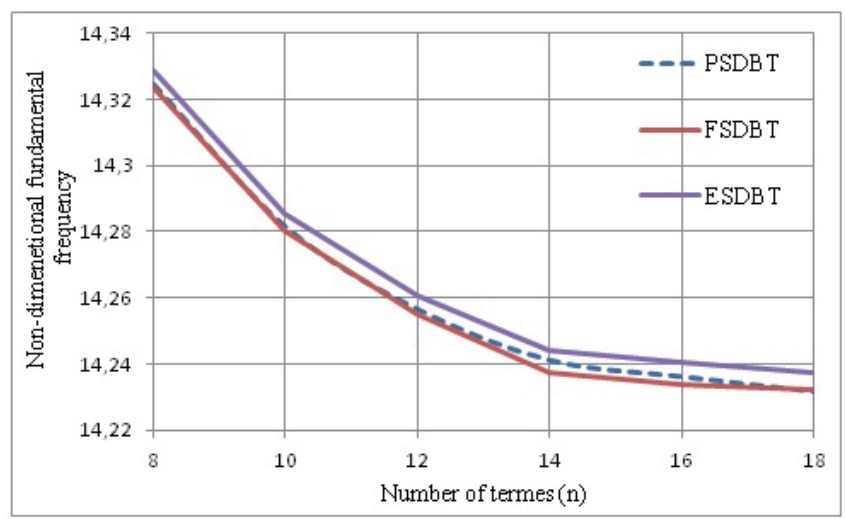

Fig. 3 Convergence study for $\mathrm{C}-\mathrm{C}$ nanobeam with $\mathrm{k}=1$ and $\mathrm{mu}=3$ for $\mathrm{L} / \mathrm{h}=10$ 
The non-dimensional fundamental frequency of S-S FG nanobeam is calculated and compared with those of Uymaz [24] and Thai [25] based on different order beams theories (CBT, FSDBT, PSDBT and ASDBT) for the nonlocal parameters $(\mu=0,2,4)$ with $k=0$. The side of FG nanobeam $\mathrm{L}$ is assumed to be $10 \mathrm{~nm}$ and Poisson's ratio $(v)$ is taken as 0.3 . Comparisons are presented in Table 4 without considering the role of Poisson's ratio in the expression of reduced stiffness coefficient $\left(Q_{11}\right)$. Good agreement has been observed for all values of comparisons.

Table 4 Comparison of non-dimensional fundamental frequency $\left(\varpi_{1}\right)$ for simply supported FG nanobeams

\begin{tabular}{llllll}
\hline$\mu\left(\mathrm{nm}^{2}\right)$ & Source & CBT & FSDBT & PSDBT & ASDBT \\
\hline \multirow{2}{*}{0} & Ref. [24] & 9.8290 & 9.7159 & 9.6938 & 9.6948 \\
& Ref. [25] & 9.8293 & 9.7075 & 9.7075 & - \\
& Present & 9.8293 & 9.7134 & 9.7138 & 9.7147 \\
\hline \multirow{2}{*}{2} & Ref. [24] & 8.9822 & 8.8791 & 8.8588 & 8.8594 \\
& Ref. [25] & 8.9826 & 8.8713 & 8.8714 & - \\
& Present & 8.9826 & 8.8769 & 8.8773 & 8.8781 \\
\hline \multirow{2}{*}{4} & Ref. [24] & 8.3222 & 8.2267 & 8.2079 & 8.2085 \\
& Ref. [25] & 8.3228 & 8.2196 & 8.2197 & - \\
& Present & 8.3228 & 8.2250 & 8.2253 & 8.2261 \\
\hline
\end{tabular}

In Tables 5-8, the effect of change of power exponent $(k)$ and nonlocal parameter $(\mu)$ on non-dimensional fundamental frequency for different boundary conditions is reported with $\mathrm{L} / \mathrm{h}=10$ using different order beam theories (CBT, FSDBT and PSDBT). These tables indicate that the maximum fundamental frequency values are obtained for $\mathrm{C}-\mathrm{C}$ support conditions. Furthermore, the lowest frequency values are obtained for $\mathrm{C}-\mathrm{F}$ support conditions.

The fundamental frequency decreases with increasing the power exponent $(\mathrm{k})$. The lowest fundamental frequency values are given by a nanobeam pure metal $(\mathrm{k} \rightarrow \infty)$ and the highest values are given by a nanobeam pure ceramic $(\mathrm{k}=0)$.

The fundamental frequency increases with the non local parameter $(\mu)$ decreasing for boundary conditions $\mathrm{C}-\mathrm{C}, \mathrm{C}-\mathrm{S}$ and $\mathrm{S}-\mathrm{S}$, as shown in Table 5 and 7; except for $\mathrm{C}-\mathrm{F}$, the fundamental frequency is slightly proportional to non local parameter $(\mu)$, as confirmed in Table 8 .
Table 5 Variation of non-dimensional fundamental frequency $\left(\varpi_{1}\right)$ of Clamped-Clamped FG nanobeam for $\mathrm{L} / \mathrm{h}=10$

\begin{tabular}{llllllll}
\hline \multirow{2}{*}{ Theory } & $\mu$ & $\mathrm{k}=0$ & $\mathrm{k}=0.5$ & $\mathrm{k}=1$ & $\mathrm{k}=5$ & $\mathrm{k}=10$ & $\begin{array}{l}\text { Full } \\
\text { metal }\end{array}$ \\
\hline & 0 & 22.8726 & 19.3912 & 17.4932 & 15.0858 & 14.5083 & 11.6462 \\
& 1 & 21.5521 & 18.2703 & 16.4795 & 14.2079 & 13.7359 & 10.9738 \\
& 2 & 20.4321 & 17.3198 & 15.6203 & 13.4647 & 13.1627 & 10.4035 \\
CBT & 3 & 19.4674 & 16.5012 & 14.8805 & 12.8249 & 12.4184 & 9.9123 \\
& 4 & 18.6254 & 15.7868 & 14.2351 & 12.2668 & 11.8789 & 9.4836 \\
& 5 & 17.8825 & 15.1566 & 13.6659 & 11.7751 & 11.3984 & 9.1053 \\
\hline & 0 & 21.6181 & 18.4319 & 16.6708 & 14.2348 & 13.6883 & 11.0065 \\
& 1 & 20.4058 & 17.3948 & 15.7297 & 13.4322 & 12.9200 & 10.3894 \\
& 2 & 19.3714 & 16.5103 & 14.9275 & 12.7482 & 12.2633 & 9.8629 \\
& 3 & 18.4760 & 15.7451 & 14.2338 & 12.1562 & 11.6944 & 9.4070 \\
& 4 & 17.6916 & 15.0750 & 13.6266 & 11.6377 & 11.1973 & 9.0077 \\
& 5 & 16.9973 & 14.4822 & 13.0895 & 11.1793 & 10.7582 & 8.6543 \\
\hline & 0 & 21.6217 & 18.4507 & 16.6753 & 14.0866 & 13.5237 & 11.0056 \\
& 1 & 20.4088 & 17.4113 & 15.7331 & 13.2957 & 12.7691 & 10.3888 \\
& 2 & 19.3740 & 16.5252 & 14.9302 & 12.6210 & 12.1243 & 9.8625 \\
& 3 & 18.4782 & 15.7587 & 14.2360 & 12.0368 & 11.5657 & 9.4068 \\
& & 17.6934 & 15.0876 & 13.6285 & 11.5250 & 11.0760 & 9.0076 \\
& 16.9990 & 14.4939 & 13.0911 & 11.0722 & 10.6425 & 8.6542 \\
\hline
\end{tabular}

Table 6 Variation of non-dimensional fundamental frequency $\left(\varpi_{1}\right)$ of Clamped-Simply FG nanobeam for $\mathrm{L} / \mathrm{h}=10$

\begin{tabular}{|c|c|c|c|c|c|c|c|}
\hline Theory & $\mu$ & $\mathrm{k}=0$ & $\mathrm{k}=0.5$ & $\mathrm{k}=1$ & $\mathrm{k}=5$ & $\mathrm{k}=10$ & $\begin{array}{l}\text { Full } \\
\text { metal }\end{array}$ \\
\hline \multirow{6}{*}{ CBT } & 0 & 15.7675 & 13.4245 & 12.2069 & 10.5824 & 10.1607 & 8.0284 \\
\hline & 1 & 14.9239 & 12.7068 & 11.5540 & 10.0161 & 9.6169 & 7.5989 \\
\hline & 2 & 14.2006 & 12.0911 & 10.9943 & 9.5305 & 9.1506 & 7.2306 \\
\hline & 3 & 13.5718 & 11.5559 & 10.5076 & 9.1084 & 8.7453 & 6.9104 \\
\hline & 4 & 13.0188 & 11.0852 & 10.0796 & 8.7372 & 8.3889 & 6.6288 \\
\hline & 5 & 12.5275 & 10.6673 & 9.6994 & 8.4075 & 8.0723 & 6.3787 \\
\hline \multirow{6}{*}{ FSDBT } & 0 & 15.2720 & 13.0400 & 11.8779 & 10.2352 & 9.7782 & 7.7611 \\
\hline & 1 & 14.4657 & 12.3513 & 11.2497 & 9.6947 & 9.2637 & 7.3515 \\
\hline & 2 & 13.7726 & 11.7592 & 10.7099 & 9.2301 & 8.8212 & 7.0006 \\
\hline & 3 & 13.1689 & 11.2436 & 10.2398 & 8.8255 & 8.4354 & 6.6939 \\
\hline & 4 & 12.6370 & 10.7893 & 9.8258 & 8.4691 & 8.0947 & 6.4241 \\
\hline & 5 & 12.1639 & 10.3856 & 9.4577 & 8.1521 & 7.7929 & 6.1838 \\
\hline \multirow{6}{*}{ PSDBT } & 0 & 15.2688 & 13.0466 & 11.8776 & 10.1750 & 9.7192 & 7.7811 \\
\hline & 1 & 14.4626 & 12.3574 & 11.2493 & 9.6390 & 9.2086 & 7.3697 \\
\hline & 2 & 13.7697 & 11.7647 & 10.7096 & 9.1781 & 8.7694 & 7.0163 \\
\hline & 3 & 13.1661 & 11.2487 & 10.2396 & 8.7764 & 8.3865 & 6.7084 \\
\hline & 4 & 12.6345 & 10.7942 & 9.8256 & 8.4224 & 8.0488 & 6.4372 \\
\hline & 5 & 12.1614 & 10.3902 & 9.4575 & 8.1075 & 7.7486 & 6.1961 \\
\hline
\end{tabular}


Table 7 Variation of non-dimensional fundamental frequency $\left(\varpi_{1}\right)$ of Simply -Simply FG nanobeam for $\mathrm{L} / \mathrm{h}=10$

\begin{tabular}{|c|c|c|c|c|c|c|c|}
\hline Theory & $\mu$ & $\mathrm{k}=0$ & $\mathrm{k}=0.5$ & $\mathrm{k}=1$ & $\mathrm{k}=5$ & $\mathrm{k}=10$ & $\begin{array}{l}\text { Full } \\
\text { metal }\end{array}$ \\
\hline \multirow{6}{*}{ CBT } & 0 & 10.1000 & 8.8090 & 8.2582 & 7.3049 & 6.8045 & 5.1427 \\
\hline & 1 & 9.6357 & 8.4039 & 7.8780 & 6.9683 & 6.4913 & 4.9063 \\
\hline & 2 & 9.2301 & 8.0499 & 7.5459 & 6.6743 & 6.2177 & 4.6997 \\
\hline & 3 & 8.8717 & 7.7372 & 7.2526 & 6.4147 & 5.9761 & 4.5172 \\
\hline & 4 & 8.5520 & 7.4584 & 6.9910 & 6.1831 & 5.7606 & 4.3545 \\
\hline & 5 & 8.2646 & 7.2076 & 6.7559 & 5.9750 & 5.5668 & 4.2081 \\
\hline \multirow{6}{*}{ FSDBT } & 0 & 9.9811 & 8.7109 & 8.1641 & 7.1987 & 6.7014 & 5.0821 \\
\hline & 1 & 9.5224 & 8.3104 & 7.7884 & 6.8671 & 6.3930 & 4.8486 \\
\hline & 2 & 9.1216 & 7.9604 & 7.4602 & 6.5775 & 6.1237 & 4.6445 \\
\hline & 3 & 8.7675 & 7.6513 & 7.1702 & 6.3216 & 5.8857 & 4.4642 \\
\hline & 4 & 8.4516 & 7.3756 & 6.9116 & 6.0935 & 5.6735 & 4.3034 \\
\hline & 5 & 8.1677 & 7.1277 & 6.6792 & 5.8884 & 5.4827 & 4.1588 \\
\hline \multirow{6}{*}{ PSDBT } & 0 & 9.9815 & 8.7127 & 8.1645 & 7.1786 & 6.6823 & 5.0824 \\
\hline & 1 & 9.5228 & 8.3121 & 7.7887 & 6.8480 & 6.3748 & 4.8488 \\
\hline & 2 & 9.1220 & 7.9621 & 7.4605 & 6.5591 & 6.1062 & 4.6447 \\
\hline & 3 & 8.7678 & 7.6528 & 7.1705 & 6.3040 & 5.8690 & 4.4644 \\
\hline & 4 & 8.4520 & 7.3771 & 6.9119 & 6.0765 & 5.6574 & 4.3035 \\
\hline & 5 & 8.1680 & 7.1291 & 6.6794 & 5.8720 & 5.4671 & 4.1589 \\
\hline
\end{tabular}

Table 8 Variation of non-dimensional fundamental frequency $\left(\varpi_{1}\right)$ of Clamped-Free $\mathrm{FG}$ nanobeam for $\mathrm{L} / \mathrm{h}=10$

\begin{tabular}{|c|c|c|c|c|c|c|c|}
\hline Theory & $\mu$ & $\mathrm{k}=0$ & $\mathrm{k}=0.5$ & $\mathrm{k}=1$ & $k=5$ & $\mathrm{k}=10$ & $\begin{array}{l}\text { Full } \\
\text { metal }\end{array}$ \\
\hline \multirow{6}{*}{ CBT } & 0 & 3.6059 & 3.0586 & 2.7614 & 2.3836 & 2.3047 & 1.8360 \\
\hline & 1 & 3.6213 & 3.0717 & 2.7732 & 2.3938 & 2.3145 & 1.8439 \\
\hline & 2 & 3.6372 & 3.0851 & 2.7854 & 2.4043 & 2.3247 & 1.8520 \\
\hline & 3 & 3.6534 & 3.0989 & 2.7978 & 2.4150 & 2.3350 & 1.8602 \\
\hline & 4 & 3.6700 & 3.1131 & 2.8106 & 2.4260 & 2.3457 & 1.8687 \\
\hline & 5 & 3.6871 & 3.1276 & 2.8237 & 2.4374 & 2.3566 & 1.8774 \\
\hline \multirow{6}{*}{ FSDBT } & 0 & 3.5858 & 3.0434 & 2.7483 & 2.3698 & 2.2898 & 1.8258 \\
\hline & 1 & 3.6004 & 3.0558 & 2.7596 & 2.3793 & 2.2989 & 1.8333 \\
\hline & 2 & 3.6155 & 3.0686 & 2.7710 & 2.3891 & 2.3084 & 1.8409 \\
\hline & 3 & 3.6308 & 3.0817 & 2.7828 & 2.3991 & 2.3180 & 1.8487 \\
\hline & 4 & 3.6466 & 3.0951 & 2.7949 & 2.4093 & 2.3279 & 1.8568 \\
\hline & 5 & 3.6628 & 3.1089 & 2.8073 & 2.4199 & 2.3381 & 1.8650 \\
\hline \multirow{6}{*}{ PSDBT } & 0 & 3.5858 & 3.0436 & 2.7484 & 2.3671 & 2.2869 & 1.8258 \\
\hline & 1 & 3.6005 & 3.0561 & 2.7596 & 2.3765 & 2.2960 & 1.8333 \\
\hline & 2 & 3.6155 & 3.0689 & 2.7711 & 2.3862 & 2.3053 & 1.8409 \\
\hline & 3 & 3.6309 & 3.0820 & 2.7829 & 2.3961 & 2.3148 & 1.8488 \\
\hline & 4 & 3.6467 & 3.0955 & 2.7951 & 2.4063 & 2.3247 & 1.8568 \\
\hline & 5 & 3.6629 & 3.1093 & 2.8075 & 2.4168 & 2.3347 & 1.8651 \\
\hline
\end{tabular}

It is also seen that the fundamental frequency calculated by the classical theory of beams (CBT) is relatively greater than those calculated by the first and high order shear deformation beam theory, whereas two latter theories (FSDBT and PSDBT) gives substantially the same frequencies when the power exponent $(k)$ take very low or very large values $(\mathrm{k} 1$ or $k \rightarrow \infty)$.

Figures 4-7 illustrate the variation of the fundamental frequency according to the material distribution $(\mathrm{k})$ for different non local parameter values $(\mu)$ under different boundary conditions with a constant slenderness ratio $\mathrm{L} / \mathrm{h}=10$. It can be observed that, the fundamental frequency decreases rapidly where the power exponent $(\mathrm{k})$ is in range from 0 to 2 , the decrease is medium for $\mathrm{k}$ in range from 2 to 5 and it is low for $\mathrm{k}$ superior to 5 at a constant non-local parameter $(\mu)$.

The influence of power exponent $(\mathrm{k})$ on the variation ratio of fundamental frequency is important for $\mathrm{C}-\mathrm{C}$ compared to the boundary conditions $\mathrm{C}-\mathrm{S}, \mathrm{S}-\mathrm{S}$ and $\mathrm{C}-\mathrm{F}$. For example, at $\mu=$ 5 as the power exponent $(\mathrm{k})$ change from 0 to 10 , the variation ratio of fundamental frequency reduced by $41.98 \%$ for $\mathrm{C}-\mathrm{C}$, $40.56 \%$ for $\mathrm{C}-\mathrm{S}, 37.06 \%$ for $\mathrm{C}-\mathrm{F}$ and $34.32 \%$ for $\mathrm{S}-\mathrm{S}$.

The effect of the nonlocality parameter is more significant when $\mu$ increase from 0 to 3 than that nonlocality parameter $(\mu)$ in interval between 3 and 5 . For example, for $\mathrm{S}-\mathrm{S}$ at $\mathrm{k}=10$, the fundamental frequency decrease with $77.11 \%$ where $\mu$ varies from 0 to 3 and $22.89 \%$ where $\mu$ varies from 3 to 5 .

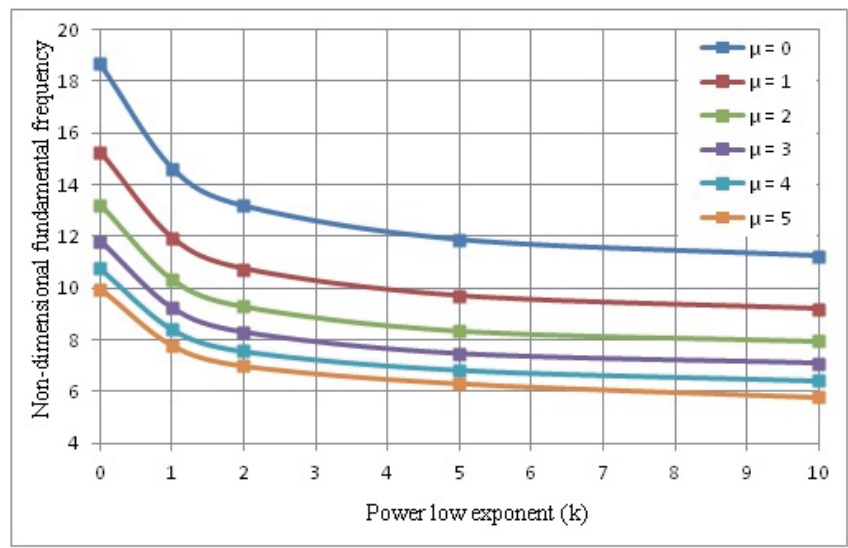

Fig. 4 Variation of non-dimensional fundamental frequency $\left(\varpi^{1}\right)$ of Clamped-Clamped FG nanobeam for $\mathrm{L} / \mathrm{h}=5$

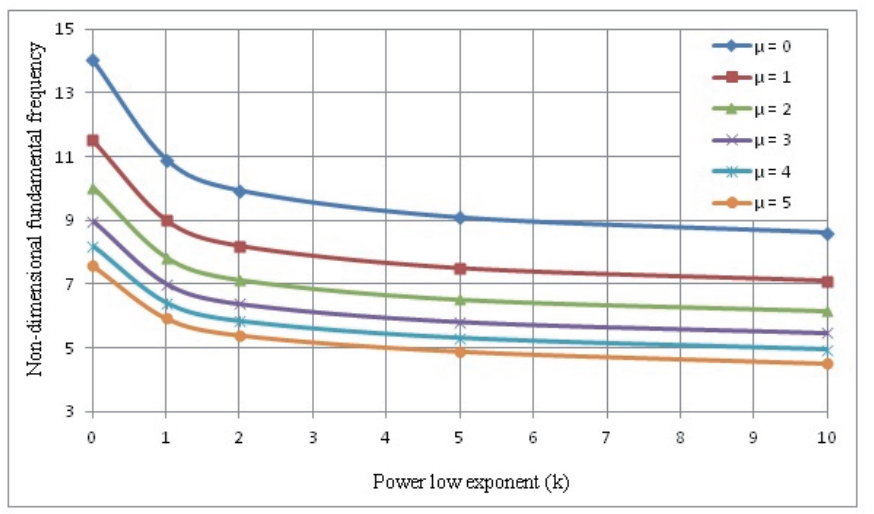

Fig. 5 Variation of non-dimensional fundamental frequency $\left(\varpi^{1}\right)$ of Clamped-Simply FG nanobeam for $\mathrm{L} / \mathrm{h}=5$ 


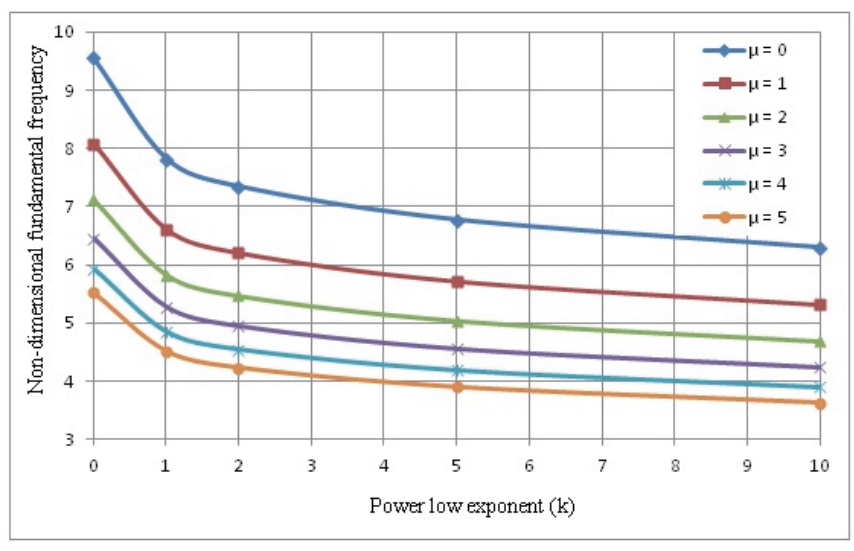

Fig. 6 Variation of non-dimensional fundamental frequency $\left(\varpi^{1}\right)$ of Simply -Simply FG nanobeam for $\mathrm{L} / \mathrm{h}=5$

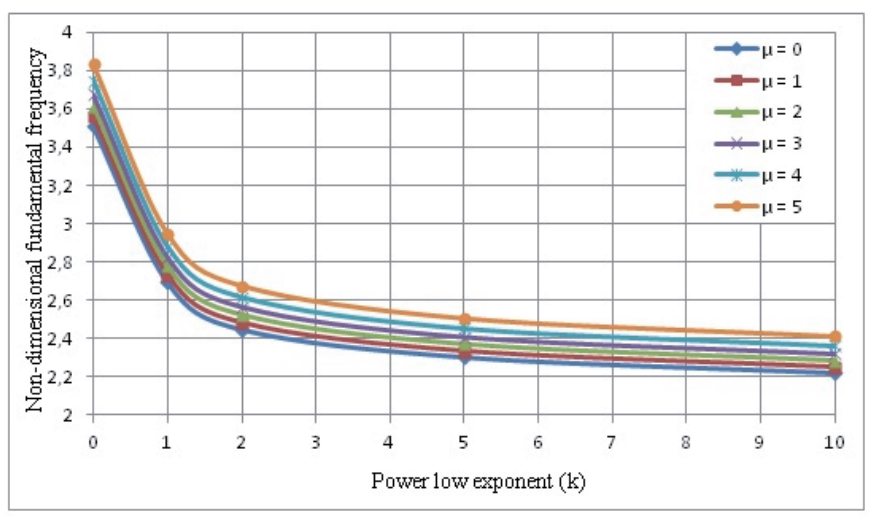

Fig. 7 Variation of non-dimensional fundamental frequency $\left(\varpi^{1}\right)$ of Clamped-Free FG nanobeam for $\mathrm{L} / \mathrm{h}=5$

Tables 9-12 show the variation of the non-dimensional fundamental frequency of $\mathrm{FG}$ nanobeams with different sets of edge supports $(\mathrm{C}-\mathrm{C}, \mathrm{C}-\mathrm{F}, \mathrm{S}-\mathrm{S}$ and $\mathrm{C}-\mathrm{S})$ while varying the non local parameter $(\mu)$ and the slenderness ratio $(\mathrm{L} / \mathrm{h})$. This variation is a function of all beams theories CBT, FSDBT, PSDBT, HSDBT, TSDBT, ESDBT and ASDBT at power exponent $\mathrm{k}=1$. It's noted that the non-dimensional fundamental frequency increase when the value of slenderness ratio $(\mathrm{L} / \mathrm{h})$ is increased for the three conditions of support C-C, C-S and S-S (Tables 9-11). Furthermore, the increase in fundamental frequency is important when the non local parameter $(\mu)$ takes superior values. For example, the difference between the fundamental frequency of $\mathrm{C}-\mathrm{S}$ FG nanobeam with $\mathrm{L} / \mathrm{h}=5$ and $\mathrm{L} / \mathrm{h}=20$ by TSDBT is $11.48 \%$ for $\mu=0,50.07 \%$ for $\mu=2$ and $89.47 \%$ for $\mu=5$.

The increase in the slenderness ratio $(\mathrm{L} / \mathrm{h})$ has different behaviors on the natural frequency of $\mathrm{C}-\mathrm{F}$ nanobeam, as observed in Table 12. It may be noted that the fundamental frequency is decreased slightly due to the increase in $\mathrm{L} / \mathrm{h}$ ratio when the nonlocal parameter $(\mu)$ is superior to 2 for different beams theories exceptionally for CBT the increase is started by $\mu=1$.

The effect of slenderness ratio $(\mathrm{L} / \mathrm{h})$ on the fundamental frequency is very important in the $\mathrm{C}-\mathrm{C}$ nanobeam case relative to other boundary conditions. For instance, the variation of the fundamental frequency FG nanobeam with L/h varying from 5 to 20 by ESDBT and $\mu=3$ is $78.75 \%$ for $\mathrm{C}-\mathrm{C}, 64.84 \%$ for C-S, $50.58 \%$ for S-S and $01.97 \%$ for C-F.

The difference between the fundamental frequency of CBT and shear deformation theories is considerable when the slenderness ratio $(\mathrm{L} / \mathrm{h})$ decreased. The fundamental frequency of the higher-order theories is a little greater than those of FSDBT for any nonlocal parameter $(\mu)$ and become almost equal when the slenderness ratio $(\mathrm{L} / \mathrm{h})$ increases. In the case $\mathrm{C}-\mathrm{F}$ nanobeam the fundamental frequency converge to a single value, (see Table 12).

Figure 8 describes the manner of variation of the fundamental frequency for ESDBT according to nonlocal parameters $(\mu)$ under different boundary conditions with $\mathrm{k}=1$ and $\mathrm{L} / \mathrm{h}=5$. It is noteworthy that, the effect of nonlocal parameters $(\mu)$ on the fundamental frequency is significant for C-C support compared to the other boundary conditions. As $\mu$ from 0 to 5 , the fundamental frequency decreased by $46.69 \%$ for C-C, $45.03 \%$ for C-S, $42.08 \%$ for S-S and increased by $09.36 \%$ for C-F.

Table 9 Non-dimensional fundamental frequency $\left(\varpi_{1}\right)$ of Clamped-Clamped FG nanobeams with $\mathrm{k}=1$

\begin{tabular}{|c|c|c|c|c|c|c|c|c|}
\hline $\mathrm{L} / \mathrm{h}$ & $\mu$ & CBT & FSDBT & PSDBT & HSDBT & TSDBT & ESDBT & ASDBT \\
\hline \multirow{6}{*}{5} & 1 & 17.2192 & 14.6324 & 14.6649 & 14.6647 & 14.6734 & 14.6927 & 14.6917 \\
\hline & 2 & 13.8454 & 11.9819 & 11.9992 & 11.9990 & 12.0039 & 12.0165 & 12.0161 \\
\hline & 3 & 11.8882 & 10.3706 & 10.3819 & 10.3818 & 10.3854 & 10.3948 & 10.3946 \\
\hline & 4 & 10.5754 & 9.2647 & 9.2732 & 9.2730 & 9.2758 & 9.2837 & 9.2835 \\
\hline & 5 & 9.6176 & 8.4475 & 8.4542 & 8.4541 & 8.4563 & 8.4633 & 8.4632 \\
\hline & 5 & 8.8796 & 7.8126 & 7.8183 & 7.8182 & 7.8203 & 7.8264 & 7.8263 \\
\hline \multirow{6}{*}{20} & 0 & 17.5730 & 17.3446 & 17.3453 & 17.3454 & 17.3456 & 17.3465 & 17.3466 \\
\hline & 1 & 17.3063 & 17.0833 & 17.0839 & 17.0840 & 17.0842 & 17.0851 & 17.0852 \\
\hline & 2 & 17.0510 & 16.8331 & 16.8336 & 16.8337 & 16.8340 & 16.8348 & 16.8349 \\
\hline & 3 & 16.8064 & 16.5933 & 16.5937 & 16.5938 & 16.5941 & 16.5949 & 16.5950 \\
\hline & 4 & 16.5717 & 16.3631 & 16.3635 & 16.3636 & 16.3639 & 16.3647 & 16.3647 \\
\hline & 5 & 16.3463 & 16.1420 & 16.1423 & 16.1424 & 16.1427 & 16.1435 & 16.1435 \\
\hline
\end{tabular}


Table 10 Non-dimensional fundamental frequency $\left(\varpi_{1}\right)$ of Clamped- Simply FG nanobeams with k=1

\begin{tabular}{|c|c|c|c|c|c|c|c|c|}
\hline $\mathrm{L} / \mathrm{h}$ & $\mu$ & CBT & FSDBT & PSDBT & HSDBT & TSDBT & ESDBT & ASDBT \\
\hline \multirow{6}{*}{5} & 0 & 12.0153 & 10.7980 & 10.9197 & 10.9189 & 10.9117 & 10.9310 & 10.8978 \\
\hline & 1 & 9.8847 & 8.9477 & 9.0461 & 9.0468 & 9.0425 & 9.0555 & 9.0342 \\
\hline & 2 & 8.5869 & 7.8131 & 7.8841 & 7.8849 & 7.8822 & 7.8922 & 7.8765 \\
\hline & 3 & 7.6926 & 6.9650 & 7.0760 & 7.0766 & 7.0748 & 7.0831 & 7.0712 \\
\hline & 4 & 7.0289 & 6.3719 & 6.4730 & 6.4737 & 6.4722 & 6.4795 & 6.4700 \\
\hline & 5 & 6.5114 & 5.8577 & 6.0012 & 6.0017 & 6.0006 & 6.0071 & 5.9994 \\
\hline \multirow{6}{*}{20} & 0 & 12.2558 & 12.1684 & 12.1689 & 12.1687 & 12.1641 & 12.1694 & 12.1693 \\
\hline & 1 & 12.0829 & 11.9973 & 11.9977 & 11.9976 & 11.9930 & 11.9982 & 11.9981 \\
\hline & 2 & 11.9169 & 11.8330 & 11.8333 & 11.8332 & 11.8288 & 11.8339 & 11.8338 \\
\hline & 3 & 11.7575 & 11.6751 & 11.6755 & 11.6754 & 11.6711 & 11.6760 & 11.6759 \\
\hline & 4 & 11.6041 & 11.5232 & 11.5236 & 11.5235 & 11.5194 & 11.5241 & 11.5240 \\
\hline & 5 & 11.4565 & 11.3770 & 11.3774 & 11.3773 & 11.3703 & 11.3779 & 11.3778 \\
\hline
\end{tabular}

Table 11 Non-dimensional fundamental frequency $\left(\varpi_{1}\right)$ of Simply - Simply FG nanobeams with $\mathrm{k}=1$

\begin{tabular}{|c|c|c|c|c|c|c|c|c|}
\hline $\mathrm{L} / \mathrm{h}$ & $\mu$ & CBT & FSDBT & PSDBT & HSDBT & TSDBT & ESDBT & ASDBT \\
\hline \multirow{6}{*}{5} & 0 & 8.1596 & 7.8221 & 7.8264 & 7.8264 & 7.8278 & 7.8303 & 7.8303 \\
\hline & 1 & 6.9044 & 6.6200 & 6.6235 & 6.6235 & 6.6247 & 6.6267 & 6.6267 \\
\hline & 2 & 6.0931 & 5.8427 & 5.8457 & 5.8457 & 5.8467 & 5.8485 & 5.8485 \\
\hline & 3 & 5.5137 & 5.2875 & 5.2901 & 5.2901 & 5.2910 & 5.2926 & 5.2926 \\
\hline & 4 & 5.0733 & 4.8654 & 4.8678 & 4.8677 & 4.8686 & 4.8701 & 4.8701 \\
\hline & 5 & 4.7240 & 4.5305 & 4.5327 & 4.5327 & 4.5335 & 4.5348 & 4.5348 \\
\hline \multirow{6}{*}{20} & 0 & 8.2834 & 8.2590 & 8.2590 & 8.2590 & 8.2591 & 8.2592 & 8.2592 \\
\hline & 1 & 8.1830 & 8.1589 & 8.1589 & 8.1589 & 8.1590 & 8.1591 & 8.1591 \\
\hline & 2 & 8.0861 & 8.0624 & 8.0624 & 8.0624 & 8.0624 & 8.0626 & 8.0626 \\
\hline & 3 & 7.9927 & 7.9692 & 7.9692 & 7.9692 & 7.9692 & 7.9694 & 7.9694 \\
\hline & 4 & 7.9023 & 7.8791 & 7.8791 & 7.8792 & 7.8792 & 7.8793 & 7.8793 \\
\hline & 5 & 7.8150 & 7.7921 & 7.7921 & 7.7921 & 7.7921 & 7.7923 & 7.7923 \\
\hline
\end{tabular}

Table 12 Non-dimensional fundamental frequency $\left(\varpi_{1}\right)$ of Clamped-Free FG nanobeams with $\mathrm{k}=1$

\begin{tabular}{|c|c|c|c|c|c|c|c|c|}
\hline $\mathrm{L} / \mathrm{h}$ & $\mu$ & CBT & FSDBT & PSDBT & HSDBT & TSDBT & ESDBT & ASDBT \\
\hline \multirow{6}{*}{5} & 0 & 2.7443 & 2.6947 & 2.6949 & 2.6949 & 2.6951 & 2.6953 & 2.6953 \\
\hline & 1 & 2.7911 & 2.7325 & 2.7336 & 2.7336 & 2.7339 & 2.7343 & 2.7343 \\
\hline & 2 & 2.8430 & 2.7747 & 2.7768 & 2.7768 & 2.7772 & 2.7778 & 2.7778 \\
\hline & 3 & 2.9014 & 2.8227 & 2.8256 & 2.8256 & 2.8262 & 2.8269 & 2.8269 \\
\hline & 4 & 2.9681 & 2.8779 & 2.8816 & 2.8816 & 2.8823 & 2.8832 & 2.8832 \\
\hline & 5 & 3.0460 & 2.9428 & 2.9472 & 2.9472 & 2.9480 & 2.9491 & 2.9491 \\
\hline \multirow{6}{*}{20} & 0 & 2.7657 & 2.7624 & 2.7624 & 2.7624 & 2.7624 & 2.7624 & 2.7624 \\
\hline & 1 & 2.7687 & 2.7653 & 2.7653 & 2.7653 & 2.7653 & 2.7653 & 2.7653 \\
\hline & 2 & 2.7717 & 2.7683 & 2.7683 & 2.7683 & 2.7683 & 2.7683 & 2.7683 \\
\hline & 3 & 2.7747 & 2.7712 & 2.7712 & 2.7712 & 2.7712 & 2.7713 & 2.7713 \\
\hline & 4 & 2.7777 & 2.7742 & 2.7742 & 2.7742 & 2.7742 & 2.7742 & 2.7742 \\
\hline & 5 & 2.7807 & 2.7772 & 2.7772 & 2.7772 & 2.7772 & 2.7772 & 2.7772 \\
\hline
\end{tabular}




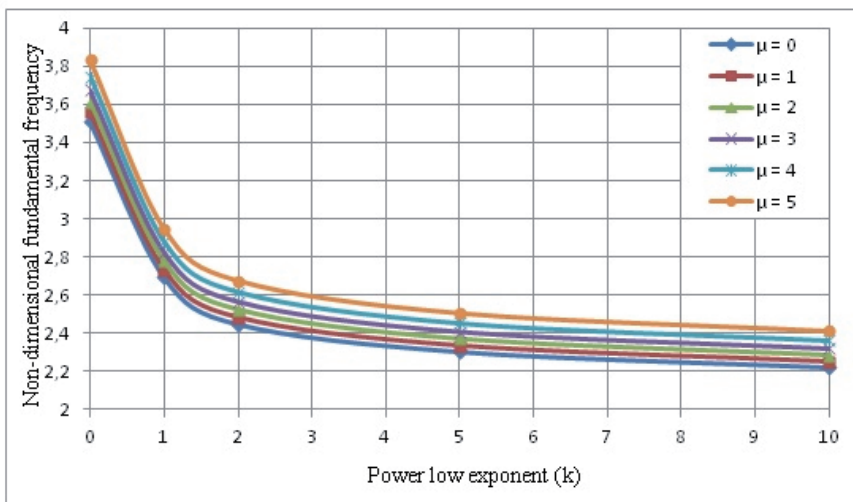

Fig. 8 Variation of non-dimensional fundamental frequency of $\mathrm{FG}$ nanobeam with nonlocal parameter at $\mathrm{L} / \mathrm{h}=5$

\section{Conclusion}

This paper has presented a free vibration analysis of FG nanobeams under different boundary conditions using various shear deformation beam theories. Based on the nonlocal differential constitutive relation of Eringen, the Ritz method is employed to solve the governing equations. The effects of material distribution, nonlocal parameter, beam theories, slenderness ratios and boundary conditions on the fundamental frequency are examined in detail. The major conclusions of this investigation is,

- In the Ritz method, the increased number (n) in the displacement functions plays a crucial role in the convergence of frequency;

- Increasing the value of the power low exponent (k) generates a decrease of the flexural rigidity.

- The influence of the power exponent, non local parameter, transverse shear deformation and slenderness ratio on the fundamental frequency value is important for FG nanobeams that present the highest stiffness;

- For short FG nanobeams, the difference between the fundamental frequency of classical beam theory and those of the first and higher-order shear deformation beam theories is considerable;

- The fundamental frequency obtained using the first order and higher-order beam theories are almost identical for the long FG nanobeams.

\section{References}

[1] Loy, C. T., Lam, K. Y., Reddy, J. N. "Vibration of functionally graded cylindrical shells." International Journal of Mechanical Sciences. 41(3), pp. 309-324. 1999. DOI: 10.1016/S0020-7403(98)00054-X

[2] Leissa, A. W. "A direct method for analyzing the forced vibrations of continuous systems having damping." Journal of Sound and Vibration. 56(3), pp. 313-324. 1978. DOI: 10.1016/S0022-460X(78)80150-3

[3] Reddy, J. N. "A simple higher-order theory for laminated composite plates." Journal of Applied Mechanics. 51(4), pp. 745-752. 1984. DOI: $10.1115 / 1.3167719$
[4] Touratier, M. "An efficient standard plate theory." International Journal of Engineering Science. 29(8), pp. 901-916. 1991. DOI: 10.1016/0020-7225(91)90165-Y

[5] Soldatos, K. P. "A transverse shear deformation theory for homogeneous monoclinic plates." Acta Mechanica. 94(3-4), pp. 195-220. 1992. DOI: $10.1007 / \mathrm{BF} 01176650$

[6] Karama, M., Afaq, K. S., Mistou, S. "Mechanical behaviour of laminated composite beam by the new multi-layered laminated composite structures model with transverse shear stress continuity." International Journal of Solids and Structures. 40(6), pp. 1525-1546. 2003. DOI: 10.1016/S0020-7683(02)00647-9

[7] Aydogdu, M. "A new shear deformation theory for laminated composite plates." Composite Structures. 89(1), pp. 94-101. 2009. DOI: 10.1016/j.compstruct.2008.07.008

[8] Eringen, A. C., Edelen, D. G. B. "On nonlocal elasticity." International Journal of Engineering Science. 10(3), pp. 233-248. 1972. DOI: 10.1016/0020-7225(72)90039-0

[9] Eringen, A. C. "Edge dislocation in nonlocal elasticity." International Journal of Engineering Science. 15(3), pp. 177-183. 1977. DOI: 10.1016/0020-7225(77)90003-9

[10] Eringen, A. C. "Vistas of nonlocal continuum physics." International Journal of Engineering Science. 30(10), pp. 1551-1565. 1992. DOI: 10.1016/0020-7225(92)90165-D

[11] Eringen, A. C. "Nonlocal Continuum Field Theories." Springer-Verlag New York, 2002. DOI: 10.1007/b97697

[12] Wang, C. M., Tan, V. B. C., Zhang, Y. Y. "Timoshenko beam model for vibration analysis of multi-walled carbon nanotubes." Journal of Sound and Vibration. 294(4-5), pp. 1060-1072. 2006. DOI: $10.1016 /$ j.jsv.2006.01.005

[13] Reddy, J. N. "Nonlocal theories for bending, buckling and vibration of beams." International Journal of Engineering Science. 45(2-8), pp. 288-307. 2007. DOI: 10.1016/j.ijengsci.2007.04.004

[14] Lu, P., Lee, H. P., Lu, C., Zhang, P. Q. "Application of nonlocal beam models for carbon nanotubes." International Journal of Solids and Structures. 44(16), pp. 5289-5300. 2007. DOI: 10.1016/j.ijsolstr.2006.12.034

[15] Reddy, J. N., Pang, S. D. "Nonlocal continuum theories of beams for the analysis of carbon nanotubes." Journal of Applied Physics. 103, pp. 1-16. 2008. DOI: 10.1063/1.2833431

[16] Aydogdu, M. "A general nonlocal beam theory: its application to nanobeam bending, buckling and vibration." Physica E: Low-dimensional Systems and Nanostructures. 41(9), pp. 1651-1655. 2009. DOI: $10.1016 /$ j.physe.2009.05.014

[17] Pradhan, S. C., Murmu, T. "Application of nonlocal elasticity and DQM in the flapwise bending vibration of a rotating nanocantilever." Physica E: Low-dimensional Systems and Nanostructures. 42(7), pp. 1944 1949. 2010. DOI: 10.1016/j.physe.2010.03.004

[18] Ansari, R., Gholami, R., Hosseini, K., Sahmani, S. "A sixth-order compact finite difference method for vibrational analysis of nanobeams embedded in an elastic medium based on nonlocal beam theory." Mathematical and Computer Modelling. 54(11-12), pp. 2577-2586. 2011. DOI: $10.1016 / \mathrm{j} . \mathrm{mcm} .2011 .06 .030$

[19] Thai, H.-T., Vo, T. P. "A nonlocal sinusoidal shear deformation beam theory with application to bending, buckling and vibration of nanobeams." International Journal of Engineering Science. 54, pp. 58-66. 2012. DOI: 10.1016/j.ijengsci.2012.01.009

[20] Eltaher, M. A., Alshorbagy, A. E., Mahmoud, F. F. "Vibration analysis of Euler-Bernoulli nanobeams by using finite element method." Applied Mathematical Modelling. 37(1), pp. 4787-4797. 2013. DOI: 10.1016/j.apm.2012.10.016 
[21] Rahmani, O., Pedram, O. "Analysis and modeling the size effect on vibration of functionally graded nanobeams based on nonlocal Timoshenko beam theory." International Journal of Engineering Science. 77, pp. 55-70. 2014. DOI: 10.1016/j.ijengsci.2013.12.003

[22] Wakashima, K., Hirano, T., Niino, M. "Space applications of advanced structural materials." ESA SP 303, 97. 1990.

[23] Simsek M., Reddy, J. N. "A unified higher order beam theory for buckling of a functionally graded microbeam embedded in elastic medium using modified couple stress theory. Composite Structures. 101, pp. 4758. 2013. DOI: 10.1016/j.compstruct.2013.01.017
[24] Uymaz, B. "Forced vibration analysis of functionally graded beams using nonlocal elasticity." Composite Structures. 105, pp. 227-239. 2013. DOI: 10.1016/j.compstruct.2013.05.006

[25] Thai, H.-T. "A nonlocal beam theory for bending, buckling, and vibration of nanobeams." International Journal of Engineering Science. 52, pp. 56-64. 2012. DOI: 10.1016/j.ijengsci.2011.11.011 